\title{
A New Approach for Solving Nonlinear Singular Boundary Value Problems*
}

\author{
Hui Zhu ${ }^{a}$, Jing $\mathrm{Niu}^{b}$, Ruimin Zhang ${ }^{a}$ and Yingzhen Lin ${ }^{a}$ \\ ${ }^{a}$ School of Applied Science and Civil Engineering, Zhuhai Campus,Beijing \\ Institute of Technology \\ 519088 Zhuhai, China \\ ${ }^{b}$ School of Mathematics and Sciences, Harbin Normal University \\ 150025 Harbin, China \\ E-mail(corresp.): njirwin@163.com
}

Received March 14, 2017; revised December 6, 2017; accepted December 6, 2017

\begin{abstract}
In this paper, an efficient method based on Quasi-Newton's method and the simplified reproducing kernel method is proposed for solving nonlinear singular boundary value problems. For the Quasi-Newton's method the convergence order is studied. The uniform convergence of the numerical solution as well as its derivatives are also proved. Numerical examples are given to demonstrate the efficiency and stability of the proposed method. The numerical results are compared with exact solutions and the outcomes of other existing numerical methods.
\end{abstract}

Keywords: nonlinear singular boundary value problem, numerical solution, Quasi-Newton's method, reproducing kernel method.

AMS Subject Classification: 34B16; 34K28; 47B32; 65L10.

\section{Introduction}

We now consider the following nonlinear singular boundary value problem with Neumann and Robin boundary conditions:

$$
\left\{\begin{array}{l}
u^{\prime \prime}(x)+\frac{\alpha}{x} u^{\prime}(x)=f(x, u(x)), \quad 0<x \leq 1, \quad \alpha \geq 1 \\
u^{\prime}(0)=0, \quad a u(1)+b u^{\prime}(1)=c
\end{array}\right.
$$

* Correspondence to: Jing Niu, School of Mathematics and Sciences, Harbin Normal University, Harbin, 150025, China.This work were supported by Youth Foundation of Heilongjiang Province (Grant No.JJ2016QN0211) and College Innovative Talent foundation of Heilongjiang Province(Grant No.UNPYSCT-2017179).

Copyright (c) 2018 The Author(s). Published by VGTU Press

This is an Open Access article distributed under the terms of the Creative Commons Attribution License (http://creativecommons.org/licenses/by/4.0/), which permits unrestricted use, distribution, and reproduction in any medium, provided the original author and source are credited. 
Here we assume nonlinear function $f(x, u)$ is continuous. $\frac{\partial f}{\partial u}$ exists and is continuous and $\frac{\partial f}{\partial u} \geq 0$ for any $0 \leq x \leq 1$.

Singular boundary value problems are frequent in a wide variety of applied mathematics and engineering practices, such as chemical reactions, gas dynamics, control and optimization theory, areas of biology and astrophysics [3,13]. Due to the difficulty of singularity, it is usually impossible to obtain the analytical solution of such equations. Hence, it's quite necessary to seek accurate numerical solutions of equation (1.1). Taylor series method is applied and the error analysis is presented in [2]. The authors [6] apply He's variational iteration method for solving equation (1.1). In [7], cubic spline method to analyze equation (1.1) is presented. The authors [1] use B-spline functions to develop a numerical method for computing approximation to the solution of equation (1.1). A new approach implementing a modified decomposition method in combination with the cubic B-spline collocation technique is introduced in [8]. Furthermore, the improved Adomian decomposition based on Green's function is discussed [18]. The finite difference method and non-polynomial cubic spline method are given in [16] and [17] respectively. Recently, a novel approach based on the operational matrix of orthonormal Bernoulli polynomial has been proposed [13]. Numerical solution of equation (1.1) based on improved differential transform method(IDTM) has also been proposed by the authors in [10]. Furthermore, the similar method based on IDTM works well for the other type of nonlinear boundary value problems [11]. Although, these numerical methods are efficient and have many advantages, a lot of computational work or a high degree of smoothness are needed.

Recently, the theory of reproducing kernel has emerged as a powerful framework in numerical analysis, differential and integral equations, and probability and statistics [5]. Based on the reproducing kernel theory, the reproducing kernel method has been used and modified by many authors $[4,9,14,15,19]$. In this work, by combining with Newton iteration and modifying the reproducing kernel method, we will find the numerical solutions of equation (1.1). It's a different approach from those previous attempts. Our method can reduce computation cost and provide highly accurate global approximate solutions.

\section{The Quasi-Newton's method}

In this section, we describe the classical Newton method which is a very effective tool for solving nonlinear problems. Consider a nonlinear equation $f(x)=0$, at the point $\left(x_{0}, f\left(x_{0}\right)\right)$,we can get a tangent equation

$$
y=f\left(x_{0}\right)+f^{\prime}\left(x_{0}\right)\left(x-x_{0}\right) .
$$

Furthermore, the equation $f(x)=0$ can be approximated by the following equation

$$
f\left(x_{0}\right)+f^{\prime}\left(x_{0}\right)\left(x-x_{0}\right)=0 .
$$

Then, a Newton's iterative formula for solving $f(x)=0$ is defined as following:

$$
x_{k+1}=x_{k}-\frac{f\left(x_{k}\right)}{f^{\prime}\left(x_{k}\right)}, \quad k=0,1,2 \ldots
$$


Now we introduce the Fréchet derivative proposed by paper [12].

Definition 1. Let $\mathcal{F}: X \rightarrow Y$, where $X$ and $Y$ are Banach spaces. Then a bounded and linear operator $\mathcal{A}: X \rightarrow Y$ is called a Fréchet derivative of $\mathcal{F}$ at $u \in X$ if

$$
\lim _{h \rightarrow 0} \frac{\|\mathcal{F}(u+h)-\mathcal{F}(u)-\mathcal{A}(h)\|}{\|h\|}=0
$$

for all $h \in X$ denoted by $\mathcal{F}^{\prime}(u)$.

Lemma 1. If $\mathcal{F}$ is a linear operator, there holds $\mathcal{F}^{\prime}(u)=\mathcal{F}$, for all $u \in X$.

Proof. Since $\mathcal{F}$ is a linear operator, we have

$$
\lim _{h \rightarrow 0} \frac{\|\mathcal{F}(u+h)-\mathcal{F}(u)-\mathcal{F}(h)\|}{\|h\|}=\lim _{h \rightarrow 0} \frac{\|\mathcal{F}(u)+\mathcal{F}(h)-\mathcal{F}(u)-\mathcal{F}(h)\|}{\|h\|}=0 .
$$

Therefore, $\mathcal{F}^{\prime}(u)=\mathcal{F}$.

Especially, we can obtain the following Lemma.

Lemma 2. If $\mathcal{F}$ is a constant operator, then $\mathcal{F}^{\prime}(u)$ is a zero operator for all $u \in X$.

Based on Fréchet derivative theory, we can get an approximate formula for equation (1.1) which is similar to formula (2.1).

Consider an operator $\mathcal{F}: C^{2}[0,1] \rightarrow C[0,1]$ as

$$
\mathcal{F}(u) \triangleq x u^{\prime \prime}+\alpha u^{\prime}+x \mathcal{N}(u),
$$

where $\mathcal{N}(u)=-f(x, u(x))$. Equation (1.1) can be rewritten as

$$
\left\{\begin{array}{l}
\mathcal{F}(u)=0, \quad 0<x \leq 1, \quad \alpha \geq 1 \\
u^{\prime}(0)=0, \quad a u(1)+b u^{\prime}(1)=c .
\end{array}\right.
$$

According the Definition (1) and Lemma (1), we can get the Fréchet derivative of $\mathcal{F}$ at $u_{0}$ :

$$
\mathcal{F}^{\prime}\left(u_{0}\right): u \mapsto x u^{\prime \prime}+\alpha u^{\prime}+x \mathcal{N}^{\prime}\left(u_{0}\right) u .
$$

Moreover the operator equation $\mathcal{F}(u)=0$ can be approximated by

$$
\mathcal{F}\left(u_{0}\right)+\mathcal{F}^{\prime}\left(u_{0}\right)\left(u-u_{0}\right)=0 .
$$

By substituting (2.2) into (2.3), we get the following linear equation

$$
x u^{\prime \prime}+\alpha u^{\prime}+x \mathcal{N}^{\prime}\left(u_{0}\right)\left(u-u_{0}\right)+x \mathcal{N}\left(u_{0}\right)=0 .
$$

Therefore, we obtain a Quasi-Newton's iterative process as the following form

$$
\left\{\begin{array}{l}
x u_{k+1}^{\prime \prime}+\alpha u_{k+1}^{\prime}+x \mathcal{N}^{\prime}\left(u_{k}\right) u_{k+1}=x \mathcal{N}^{\prime}\left(u_{k}\right) u_{k}-x \mathcal{N}\left(u_{k}\right) \\
u_{k}^{\prime}(0)=0, \quad a u_{k}(1)+b u_{k}^{\prime}(1)=c, \quad k=0,1,2 \ldots
\end{array}\right.
$$


and its general form

$$
\mathcal{F}\left(u_{k}\right)+\mathcal{F}^{\prime}\left(u_{k}\right)\left(u_{k+1}-u_{k}\right)=0 .
$$

It can be seen equation (2.4) and equation (2.5) are linear equations. We could get the numerical solution. Firstly we choose a simple polynomial function satisfying the boundary conditions as an initial function $u_{0}$. Then by substituting $u_{0}$ into equation (2.4), we can get a solution $u_{1}$. And after several rounds of iteration, a high accuracy numerical result can be obtained. Next, we analyze the the convergence of our iterative algorithm.

Theorem 1. Let Fréchet derivative $\mathcal{F}^{\prime}$ and $\mathcal{N}$ be operators mentioned in equation (2.4) and equation (2.5). Assume that $\left\|\mathcal{F}^{\prime-1}\right\|\left\|\mathcal{N}^{\prime \prime}\right\|=\gamma<1$. Further, the initial function $u_{0}$ satisfies that $\left\|u_{0}-u\right\|<1$, where $u$ is the exact solution of equation (1.1). Then, iterative formula equation (2.4) or equation (2.5) is convergence, and the absolute error satisfies $\left|u_{k}-u\right| \leq\left\|u_{k}-u\right\| \leq \gamma^{2^{k}-1}$.

Proof. Based on equation (2.5), we have

$$
u_{k+1}-u_{k}=-\left[\mathcal{F}^{\prime}\left(u_{k}\right)\right]^{-1} \mathcal{F}\left(u_{k}\right) .
$$

Supposing $u(x)$ is a solution of $\mathcal{F}(u)=0$, and $E_{k}(x)=u_{k}(x)-u(x)$. Then,

$$
E_{k+1}=E_{k}-\left[\mathcal{F}^{\prime}\left(u_{k}\right)\right]^{-1}\left(\mathcal{F}\left(u_{k}\right)-\mathcal{F}(u)\right) .
$$

Thus,

$$
\mathcal{F}^{\prime}\left(u_{k}\right) E_{k+1}=\mathcal{F}^{\prime}\left(u_{k}\right) E_{k}-\left(\mathcal{F}\left(u_{k}\right)-\mathcal{F}(u)\right)
$$

Note that

$$
\begin{aligned}
& \mathcal{F}^{\prime}\left(u_{k}\right) E_{k}=x E_{k}^{\prime \prime}+\alpha E_{k}^{\prime}+x \mathcal{N}^{\prime}\left(u_{k}\right) E_{k} \\
& \mathcal{F}\left(u_{k}\right)-\mathcal{F}(u)=x u_{k}^{\prime \prime}+\alpha u_{k}^{\prime}+x \mathcal{N}\left(u_{k}\right)-\left(x u^{\prime \prime}+\alpha u^{\prime}+x \mathcal{N}(u)\right) \\
& \quad=x E_{k}^{\prime \prime}+\alpha E_{k}^{\prime}+x\left(\mathcal{N}\left(u_{k}\right)-\mathcal{N}(u)\right)=x E_{k}^{\prime \prime}+\alpha E_{k}^{\prime}+x \mathcal{N}^{\prime}\left(\xi_{k}\right) E_{k}
\end{aligned}
$$

Substituting (2.7), (2.8) into (2.6), we obtain

$$
\begin{aligned}
\mathcal{F}^{\prime}\left(u_{k}\right) E_{k+1} & =x E_{k}^{\prime \prime}+\alpha E_{k}^{\prime}+x \mathcal{N}^{\prime}\left(u_{k}\right) E_{k}-\left(x E_{k}^{\prime \prime}+\alpha E_{k}^{\prime}+x \mathcal{N}^{\prime}\left(\xi_{k}\right) E_{k}\right) \\
& =x\left(\mathcal{N}^{\prime}\left(u_{k}\right)-\mathcal{N}^{\prime}\left(\xi_{k}\right)\right) E_{k}=x \mathcal{N}^{\prime \prime}\left(\eta_{k}\right)\left(u_{k}-\xi_{k}\right) E_{k} .
\end{aligned}
$$

As $\left|u_{k}-\xi_{k}\right| \leq\left|u_{k}-u\right|=\left|E_{k}\right|$, we have

$$
\left\|E_{k+1}\right\| \leq\left\|\mathcal{F}^{\prime}\left(u_{k}\right)^{-1}\right\|\left\|\mathcal{N}^{\prime \prime}\left(\eta_{k}\right)\right\|\left\|E_{k}\right\|^{2}<\gamma\left\|E_{k}\right\|^{2} .
$$

Furthermore, we discuss the convergence order based on the mathematical induction. Firstly,

$$
\left\|E_{0}\right\|=\left\|u_{0}-u\right\|<1=\gamma^{2^{0}-1} .
$$

Let

$$
\left\|E_{k}\right\|=\left\|u_{k}-u\right\|=\gamma^{2^{k}-1},
$$

Hence,

$$
\left\|E_{k+1}\right\|<\gamma\left\|E_{k}\right\|^{2}=\gamma\left(\gamma^{2^{k}-1}\right)^{2}=\gamma^{2^{k+1}-1} .
$$

Thus, the proof is completed. 


\section{The simplified reproducing kernel method}

In this section, we focus on solving the linear equation by simplified reproducing kernel method. For convenience, we discuss the simplified equation of equation (2.4) as follows

$$
\left\{\begin{array}{l}
x u^{\prime \prime}(x)+\alpha u^{\prime}(x)+x a_{2}(x) u=h(x), \\
u^{\prime}(0)=0, \quad a u(1)+b u^{\prime}(1)=c,
\end{array}\right.
$$

where $a_{2}(x)=\mathcal{N}^{\prime}\left(u_{k}\right), h(x)=x\left(\mathcal{N}^{\prime}\left(u_{k}\right) u_{k}-\mathcal{N}\left(u_{k}\right)\right)$.

Now, we introduce the reproducing kernel method for equation (3.1). From [4], it follows that $W_{2}^{3}[0,1], W_{2}^{1}[0,1]$ are reproducing kernel spaces with reproducing kernel functions $R_{t}(x)$ and $r_{t}(x)$ respectively. Considering an operator $\mathcal{L}: W_{2}^{3}[0,1] \rightarrow W_{2}^{1}[0,1]$,

$$
\mathcal{L} u=x u^{\prime \prime}(x)+\alpha u^{\prime}(x)+x a_{2}(x) u(x),
$$

equation (3.1) can be written as

$$
\left\{\begin{array}{l}
\mathcal{L} u=h(x), \\
u^{\prime}(0)=0, \quad a u(1)+b u^{\prime}(1)=c .
\end{array}\right.
$$

Lemma 3. Let $\mathcal{L}^{*}$ be a conjugate operator of $\mathcal{L}$, then $\left(\mathcal{L}^{*} r_{t}\right)(x)=\left(\mathcal{L} R_{x}\right)(t)$.

Taking a dense set $\left\{x_{i}\right\}_{i=1}^{\infty}$ on $[0,1]$, we get a function system $\left\{\psi_{i}(x)\right\}_{i=1}^{\infty}$ in $W_{2}^{3}$, where $\psi_{i}(x)=\mathcal{L}^{*} r_{x}\left(x_{i}\right)$.

Theorem 2. $\left\{\psi_{i}(x)\right\}_{i=1}^{n}$ is linear independent.

For the proof, one may refer to [4].

Let

$$
\begin{aligned}
& \varphi_{1}(x)=\left.\frac{\partial R_{t}(x)}{\partial t}\right|_{t=0}, \quad \varphi_{2}(x)=a R_{1}(x)+\left.b \frac{\partial R_{t}(x)}{\partial t}\right|_{t=1}, \\
& S_{n+2}=\operatorname{span}\left\{\left\{\psi_{i}\right\}_{1}^{n} \cup \varphi_{1} \cup \varphi_{2}\right\}, \quad S=\overline{\bigcup_{i=1}^{\infty} S_{i+2}} .
\end{aligned}
$$

Then $S_{n+2} \subset S \subset W_{2}^{3}$.

Theorem 3. Let $u$ be the exact solution of the operator equation (3.2). Then $\mathcal{P} u$ is also the solution of (3.2), where $\mathcal{P}$ is the orthogonal project operator from $W_{2}^{3}$ to $S$.

Let $\mathcal{P}_{n+2}$ is the orthogonal project operator from $W_{2}^{3}$ to $S_{n+2}$.

Theorem 4. $\mathcal{P}_{n+2} u$ is the solution of the following linear equation system

$$
\left\{\begin{array}{l}
\left\langle v, \psi_{i}\right\rangle=h\left(x_{i}\right), \quad i=1,2, \ldots n \\
\left\langle v, \varphi_{1}\right\rangle=0, \quad\left\langle v, \varphi_{2}\right\rangle=c .
\end{array}\right.
$$


Proof.

$$
\begin{gathered}
\left\langle\mathcal{P}_{n+2} u(\cdot), \psi_{i}(\cdot)\right\rangle=\left\langle u(\cdot), \mathcal{P}_{n+2} \psi_{i}(\cdot)\right\rangle=\left\langle u(\cdot), \psi_{i}(\cdot)\right\rangle=\left\langle u(\cdot), \mathcal{L}^{*} r_{x_{i}}(\cdot)\right\rangle \\
=\left\langle\mathcal{L} u(\cdot), r_{x_{i}}(\cdot)\right\rangle=\left\langle h(\cdot), r_{x_{i}}(\cdot)\right\rangle=h\left(x_{i}\right), \\
\left\langle\mathcal{P}_{n+2} u(\cdot), \varphi_{1}(\cdot)\right\rangle=\left\langle u(\cdot), \mathcal{P}_{n+2} \varphi_{1}(\cdot)\right\rangle=\left\langle u(\cdot), \varphi_{1}(\cdot)\right\rangle=\left\langle u(\cdot),\left.\frac{\partial R_{x}(\cdot)}{\partial x}\right|_{x=0}\right\rangle \\
=\left.\frac{\partial}{\partial x}\left\langle u(\cdot), R_{x}(\cdot)\right\rangle\right|_{x=0}=\left.\frac{\partial}{\partial x} u(x)\right|_{x=0}=u^{\prime}(0)=0 \\
\left\langle\mathcal{P}_{n+2} u(\cdot), \varphi_{2}(\cdot)\right\rangle=\left\langle u(\cdot), \mathcal{P}_{n+2} \varphi_{2}(\cdot)\right\rangle=\left\langle u(\cdot), \varphi_{2}(\cdot)\right\rangle \\
=\left\langle u(\cdot), a R_{1}(\cdot)+\left.b \frac{\partial R_{x}(\cdot)}{\partial x}\right|_{x=1}\right\rangle=a u(1)+\left.b \frac{\partial}{\partial x}\left\langle u(\cdot), R_{x}(\cdot)\right\rangle\right|_{x=1} \\
=a u(1)+\left.b \frac{\partial}{\partial x} u(x)\right|_{x=1}=a u(1)+b u^{\prime}(1)=c .
\end{gathered}
$$

Denote $u_{n}=\mathcal{P}_{n+2} u$. It can be shown that $u_{n}$ is an approximate solution of $u$. Furthermore, we can prove the uniform convergence.

Theorem 5. $u_{n}^{(i)}(x)$ uniformly converge to $u^{(i)}(x)$ on $[0,1], i=0,1,2$.

Proof.

$$
\begin{aligned}
& \left|u_{n}^{(i)}(x)-u^{(i)}(x)\right|=\left|\left\langle u_{n}(\cdot)-u(\cdot), R_{x}(\cdot)\right\rangle^{(i)}\right| \\
& \quad=\left|\left\langle u_{n}(\cdot)-u(\cdot), \frac{\partial^{i}}{\partial x^{i}} R_{x}(\cdot)\right\rangle\right| \leq\left\|u_{n}-u\right\|\left\|\mid \frac{\partial^{i} R_{x}}{\partial x^{i}}\right\| .
\end{aligned}
$$

Note that $\left\|\frac{\partial^{i} R_{x}}{\partial x^{i}}\right\|$ is bounded on $[0,1]$, thus

$$
\left|u_{n}^{(i)}(x)-u^{(i)}(x)\right| \leq M\left\|u_{n}-u\right\| \rightarrow 0 .
$$

The proof is completed.

Hence, $u_{n}$ is a good approximate solution of (3.2). Since $u_{n} \in S_{n+2}$, we get

$$
u_{n}=\sum_{i=1}^{n} \alpha_{i} \psi_{i}+\beta_{1} \varphi_{1}+\beta_{2} \varphi_{2} .
$$

As $u_{n}$ is the solution of equation (3.3), we have

$$
\left\{\begin{array}{l}
\sum_{j=1}^{n} \alpha_{j}\left\langle\psi_{j}, \psi_{i}\right\rangle+\beta_{1}\left\langle\varphi_{1}, \psi_{i}\right\rangle+\beta_{2}\left\langle\varphi_{2}, \psi_{i}\right\rangle=h\left(x_{i}\right), \quad i=1,2, \ldots n, \\
\sum_{j=1}^{n} \alpha_{j}\left\langle\psi_{j}, \varphi_{1}\right\rangle+\beta_{1}\left\langle\varphi_{1}, \varphi_{1}\right\rangle+\beta_{2}\left\langle\varphi_{2}, \varphi_{1}\right\rangle=0 \\
\sum_{j=1}^{n} \alpha_{j}\left\langle\psi_{j}, \varphi_{2}\right\rangle+\beta_{1}\left\langle\varphi_{1}, \varphi_{2}\right\rangle+\beta_{2}\left\langle\varphi_{2}, \varphi_{2}\right\rangle=c .
\end{array}\right.
$$

The coefficients of formula (3.4) are the solution of linear equation system (3.5), hence we get the approximate solution $u_{n}$ of equation (3.1). 


\section{Numerical examples}

In this section, numerical examples are tested to demonstrate the efficiency and stability of our method. All examples are computed by Mathematica 7.0.

Example 1. Consider the nonlinear differential equation $[1,18]$ :

$$
\left\{\begin{array}{l}
u^{\prime \prime}(x)+\frac{1}{x} u^{\prime}(x)=-e^{u(x)}, \quad 0<x<1, \\
u^{\prime}(0)=0, u(1)=0 .
\end{array}\right.
$$

The exact solution is $u(x)=2 \ln \left(\frac{4-2 \sqrt{2}}{(3-2 \sqrt{2}) x^{2}+1}\right)$. In our method, we need to take a group of parameters, such as initial function $u_{0}(x)$, the number of iterations $k$, and the number of dense points $n$. Let $u_{0}(x)=x^{4}$. The absolute error function is $E=\left|u_{k, n}(x)-u(x)\right|, 0<x \leq 1$, where $u_{k, n}(x)$ is the approximate solution. Table 1 compares the results for this example by the proposed method and the methods in $[1,18]$.

Table 1. Maximum absolute error for Example 1

\begin{tabular}{ccccccc}
\hline$k$ & $n$ & $E$ & $n$ & $E$ in $[18]$ & $n$ & $E$ in $[1]$ \\
\hline 3 & $\mathrm{n}=4$ & $5.67 \mathrm{E}-05$ & 12 & $4.01 \mathrm{E}-04$ & 20 & $3.16 \mathrm{E}-05$ \\
3 & $\mathrm{n}=6$ & $3.14 \mathrm{E}-06$ & 16 & $2.54 \mathrm{E}-05$ & 40 & $7.87 \mathrm{E}-06$ \\
3 & $\mathrm{n}=8$ & $6.35 \mathrm{E}-07$ & 20 & $2.10 \mathrm{E}-06$ & 60 & $3.50 \mathrm{E}-06$ \\
\hline
\end{tabular}

Figure 1 shows comparison of exact and approximate solution for $k=3$, $n=8$ and also plots the absolute errors for $k=3, n=4,6,8$. We can see that the presented method converges rapidly to the exact solution by iterating only three times.
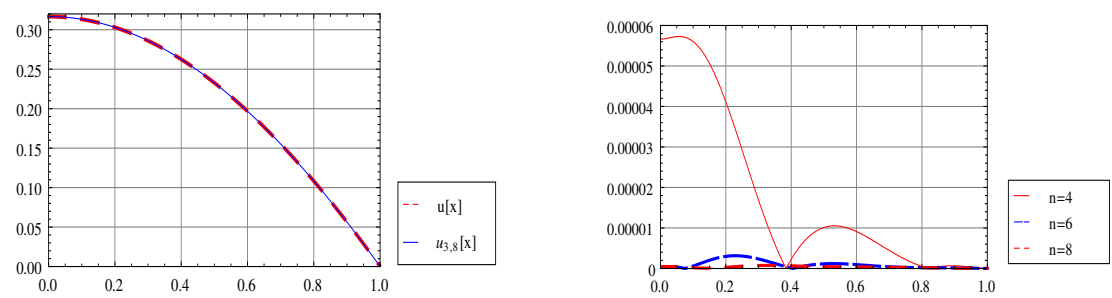

Figure 1. Comparison of exact solution $u(x)$ and approximate solution $u_{3,8}(x)$ (left side) and $E$ (right side) for Example $1(k=3)$.

Example 2. Consider the nonlinear singular boundary value problem describing the equilibrium of the isothermal gas sphere $[3,10,18]$

$$
\left\{\begin{array}{l}
u^{\prime \prime}(x)+\frac{2}{x} u^{\prime}(x)=-u^{5}(x), \quad 0<x<1, \\
u^{\prime}(0)=0, \quad u(1)=\sqrt{3 / 4}
\end{array}\right.
$$


The exact solution is $u(x)=\sqrt{3 /\left(3+x^{2}\right)}$. Let $u_{0}(x)=x^{2}$. Table 2 shows the comparisons of the presented method and the method used in $[3,10,18]$. After three iterations, the maximal absolute error can reach $10^{-7}$ at $n=10$. We have higher accuracy compared to the other mentioned methods.

Figure 2 gives comparison of $u(x)$ and $u_{4,10}(x)$ and also shows the absolute errors for $k=3, n=4,6,10$. It indicates that the curves of the exact solution and the approximate solution are overlap. The approximate solution is getting more and more accurate as the number of dense points $n$ increases. Our approach has good convergence and precision for numerical calculation.

Table 2. Maximum absolute error for Example 2

\begin{tabular}{ccccccccc}
\hline$k$ & $n$ & $E$ & $n$ & $E$ in [18] & $n$ & $E$ in [3] & $n$ & $E$ in [10] \\
\hline 4 & $n=4$ & $3.25 \mathrm{E}-05$ & 12 & $1.40 \mathrm{E}-03$ & 16 & $3.64 \mathrm{E}-04$ & 12 & $1.68 \mathrm{E}-04$ \\
4 & $n=6$ & $6.74 \mathrm{E}-06$ & 16 & $2.47 \mathrm{E}-04$ & 32 & $2.49 \mathrm{E}-05$ & 16 & $1.65 \mathrm{E}-05$ \\
4 & $n=10$ & $7.30 \mathrm{E}-07$ & 20 & $4.86 \mathrm{E}-05$ & 64 & $1.60 \mathrm{E}-06$ & 20 & $1.66 \mathrm{E}-06$ \\
\hline
\end{tabular}

Figure 2 gives comparison of $u(x)$ and $u_{4,10}(x)$ and also shows the absolute errors for $k=3, n=4,6,10$. It indicates that the curves of the exact solution and the approximate solution are overlap. The approximate solution is getting more and more accurate as the number of dense points $n$ increases. Our approach has good convergence and precision for numerical calculation.
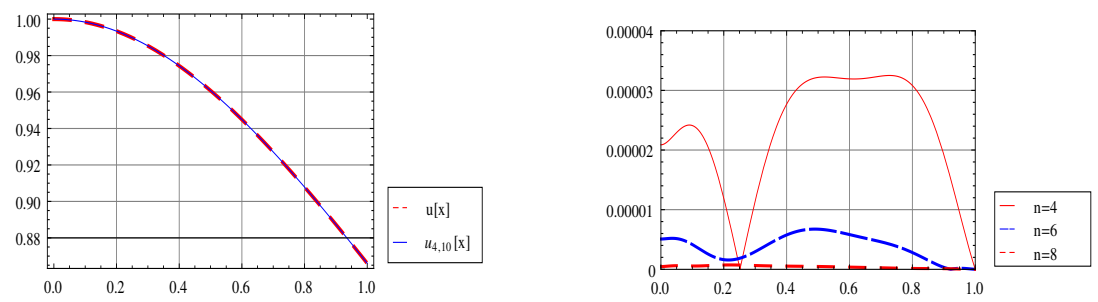

Figure 2. Comparison of exact solution $u(x)$ and approximate solution $u_{4,10}(x)$ (left side) and $E$ (right side) for Example $2(k=4)$.

Example 3. Consider the nonlinear singular boundary value problem studying the distribution of heat sources in the human head $[7,10,13,16]$.

$$
\left\{\begin{array}{l}
u^{\prime \prime}(x)+\frac{2}{x} u^{\prime}(x)=-e^{-u(x)}, \quad 0<x \leq 1 \\
u^{\prime}(0)=0, \quad u(1)+u^{\prime}(1)=0 .
\end{array}\right.
$$

Let $u_{0}(x)=x^{3}$. Since the exact solution of equation (4.1) is not known,we calculate function values of approximate solution in ten points which are presented in $[7,10,13,16]$. The numerical results for $k=3, n=10$ are listed in Table 3 . The proposed method is in good agreement to other methods $[7,10,13,16]$. 
Table 3. Numerical results for Example 3

\begin{tabular}{llllll}
\hline$x$ & Method in [16] & Method in [7] & Method in [13] & Method in [10] & Present Method \\
\hline 0 & 0.3675169710 & 0.3675179806 & 0.3675152742 & 0.3675167997 & 0.3675166189 \\
0.1 & 0.3663623697 & 0.3663634922 & 0.3663623292 & 0.3663623137 & 0.3663622527 \\
0.2 & 0.3628941066 & 0.3628952219 & 0.3628940661 & 0.3628940507 & 0.3628940189 \\
0.3 & 0.3570975862 & 0.3570986892 & 0.3570975457 & 0.3570975301 & 0.3570975087 \\
0.4 & 0.3489484612 & 0.3489495463 & 0.3489484206 & 0.3489484049 & 0.3489483815 \\
0.5 & 0.3384121893 & 0.3384132503 & 0.3384121487 & 0.3384121330 & 0.3384121172 \\
0.6 & 0.3254435631 & 0.3254445926 & 0.3254435224 & 0.3254435063 & 0.3254434945 \\
0.7 & 0.3099860810 & 0.3099870706 & 0.3099860402 & 0.3099860240 & 0.3099860196 \\
0.8 & 0.2919711440 & 0.2919720836 & 0.2919711030 & 0.2919710864 & 0.2919711009 \\
0.9 & 0.2713170512 & 0.2713179290 & 0.2713170101 & 0.2713169936 & 0.2713170010 \\
1.0 & 0.2479277646 & 0.2479285660 & 0.2479277233 & 0.2479277073 & 0.2479277424 \\
\hline
\end{tabular}

Example 4. Consider the nonlinear singular boundary value problem arising in the study of steady-state oxygen diffusion in a spherical cell $[1,10,13,17]$.

$$
\left\{\begin{array}{l}
u^{\prime \prime}(x)+\frac{\alpha}{x} u^{\prime}(x)=\frac{\delta u(x)}{u(x)+\mu}, \quad \delta>0, \mu>0, \\
u^{\prime}(0)=0, \quad 5 u(1)+5 u^{\prime}(1)=5
\end{array}\right.
$$

where $\delta$ and $\mu$ are often taken as 0.76129 and 0.03119 , respectively. And we take the value of $\alpha$ as 2. This equation does not have exact solution. Instead, we discuss the absolute residual error function which is a measure of how well the approximation satisfies the equation (4.2) as

$$
R=\left|u_{k, n}^{\prime \prime}+\frac{\alpha}{x} u_{k, n}^{\prime}-\frac{\delta u_{k, n}}{u_{k, n}+\mu}\right|, \quad 0<x \leq 1 .
$$

Taking $u_{0}(x)=x^{3}$, a good approximation is obtained for $k=4, n=10$. The numerical results of different methods are listed in Table 4.

Table 4. Numerical results for Example 4

\begin{tabular}{lccccc}
\hline$x$ & Method in [17] & Method in [1] & Method in [13] & Method in [10] & Present Method \\
\hline 0 & 0.8284833089 & 0.8284832730 & 0.8284832819 & 0.8284832870 & 0.8284833754 \\
0.1 & 0.8297060779 & 0.8297060752 & 0.8297060924 & 0.8297060890 & 0.8297061155 \\
0.2 & 0.8333747471 & 0.8333747169 & 0.8333747336 & 0.8333747303 & 0.8333747353 \\
0.3 & 0.8394899001 & 0.8394898981 & 0.8394899140 & 0.8394899106 & 0.8394899068 \\
0.4 & 0.8480527729 & 0.8480527704 & 0.8480527850 & 0.8480527816 & 0.8480527759 \\
0.5 & 0.8590649189 & 0.8590649140 & 0.8590649272 & 0.8590649239 & 0.8590649167 \\
0.6 & 0.8725283096 & 0.8725283084 & 0.8725283200 & 0.8725283166 & 0.8725283100 \\
0.7 & 0.8884452969 & 0.8884452959 & 0.8884453056 & 0.8884453023 & 0.8884452975 \\
0.8 & 0.9068185417 & 0.9068185403 & 0.9068185481 & 0.9068185448 & 0.9068185422 \\
0.9 & 0.9276509838 & 0.9276509825 & 0.9276509884 & 0.9276509853 & 0.9276509849 \\
1.0 & 0.9509457969 & 0.9509457946 & 0.9509457985 & 0.9509457960 & 0.9509457969 \\
\hline
\end{tabular}

Table 5 describes the comparison of absolute residual error between presented method and IDTM [10]. The implementation of our approach in good line with existing methods $[1,10,13,17]$ has a fast convergence speed and high accuracy. 
Table 5. Maximum absolute residual error for Example 4

\begin{tabular}{ccccc}
\hline$k$ & $n$ & $R$ & $n$ & $R$ in $[10]$ \\
\hline 4 & $n=4$ & $4.25 \mathrm{E}-05$ & 4 & $4.84 \mathrm{E}-04$ \\
4 & $n=6$ & $6.14 \mathrm{E}-06$ & 6 & $6.78 \mathrm{E}-05$ \\
4 & $n=8$ & $1.38 \mathrm{E}-06$ & 8 & $9.45 \mathrm{E}-06$ \\
4 & $n=10$ & $8.39 \mathrm{E}-07$ & 10 & $1.31 \mathrm{E}-06$ \\
\hline
\end{tabular}

\section{Conclusions}

To summarize, in this paper, we propose a new approach for solving nonlinear singular boundary value problem. First, we reduce the nonlinear problem to a linear problem based on the Quasi-Newton's method. And second, the simplified reproducing kernel method which has less computation than before is presented to solve the linear problem. The approximate solution can be obtained after iterative computation. Our method is tested by four examples arising in applied science. The numerical results show that our method has higher accuracy, less calculation and high efficiency. It is worthy to note that the approach detailed here can be readily adapted to the case of nonlinear integral and integro-differential equation with various boundary value conditions.

\section{References}

[1] H. Çă̆lar, N. Çağlar and M. Özer. B-spline solution of non-linear singular boundary value problems arising in physiology. Chaos Solitons and Fractals, 39(3):1232-1237, 2009. https://doi.org/10.1016/j.chaos.2007.06.007.

[2] S.H. Chang. Taylor series method for solving a class of nonlinear singular boundary value problems arising in applied science. Applied Mathematics and Computation, 235:110-117, 2014. https://doi.org/10.1016/j.amc.2014.02.094.

[3] M. Chawla, R. Subramanian and H. Sathi. A fourth order method for a singular two-point boundary value problem. BIT, 28(1):88-97, 1988. https://doi.org/10.1007/BF01934697.

[4] M.G. Cui. Nonlinear Numerical Analysis in the Reproducing Kernel Space. Nova Science, NewYork, 2009.

[5] A. Daniel. Reproducing Kernel Spaces and Applications. Springer, NewYork, 2013.

[6] A.S.V. Ravi Kanth and K. Aruna. He's variational iteration method for treating nonlinear singular boundary value problems. Computers and Mathematics with Applications, 60(3):821-829, 2010. https://doi.org/10.1016/j.camwa.2010.05.029.

[7] A.S.V. Ravi Kanth and V. Bhattacharya. Cubic spline for a class of non-linear singular boundary value problems arising in physiology. Applied Mathematics and Computation, 174(1):768-774, 2006. https://doi.org/10.1016/j.amc.2005.05.022.

[8] S.A. Khuri and A. Sayfy. A novel approach for the solution of a class of singular boundary value problems arising in physiology. Mathematical and Computer Modelling, 52(3-4):626-636, 2010. https://doi.org/10.1016/j.mcm.2010.04.009. 
[9] Y.Z. Lin, J. Niu and M.G. Cui. A numerical solution to nonlinear second order three-point boundary value problems in the reproducing kernel space. Applied Mathematics and Computation, 218(14):7362-7368, 2012. https://doi.org/10.1016/j.amc.2011.11.009.

[10] L.J.Xie, C.L.Zhou and S.Xu. An effective numerical method to solve a class of nonlinear singular boundary value problems using improved differential transform method. SpringerPlus, 5:1066-1084, 2016. https://doi.org/10.1186/s40064016-2753-9.

[11] L.J.Xie, C.L.Zhou and S.Xu. A new algorithm based on differential transform method for solving multi-point boundary value problems. International Journal of Computer Mathematics, 93(6):981-994, 2016. https://doi.org/10.1080/00207160.2015.1012070.

[12] P.R. Mcgilliuray and D.W. Olenburg. Methods for calculating Frechet derivatives and senstivities for the non-linear inversion problem. Geophysical Prospecting, 38(5):499-524, 1990. https://doi.org/10.1111/j.1365-2478.1990.tb01859.x.

[13] M. Mohsenyzadeh, K. Maleknejad and R. Ezzati. A numerical approach for the solution of a class of singular boundary value problems arising in physiology. Advances in Difference Equations, 231(1):231, 2015. https://doi.org/10.1186/s13662-015-0572-x.

[14] J. Niu, Y.Z. Lin and C.P. Zhang. Approximate solution of nonlinear multi-point boundary value problem on the half-line. Mathematical Modelling and Analysis, 17(2):190-202, 2012. https://doi.org/10.3846/13926292.2012.660889.

[15] J. Niu, Y.Z. Lin and C.P. Zhang. Numerical solution of nonlinear three-point boundary value problem on the positive half-line. Mathematical Methods in the Applied Sciences, 35:1601-1610, 2012. https://doi.org/10.1002/mma.2549.

[16] R.K. Pandey and Arvind K.Singh. On the convergence of a finite difference method for a class of singular boundary value problems arising in physiology. Journal of Computational and Applied Mathematics, 166(2):553-564, 2004. https://doi.org/10.1016/j.cam.2003.09.053.

[17] J. Rashidinia, R. Mohammadi and R. Jalilian. The numerical solution of non-linear singular boundary value problems arising in physiology. Applied Mathematics and Computation, 185(1):360-367, 2007. https://doi.org/10.1016/j.amc.2006.06.104.

[18] R. Singh and J. Kumar. An efficient numerical technique for the solution of nonlinear singular boundary value problems. Computer Physics Communications, 185(4):1282-1289, 2014. https://doi.org/10.1016/j.cpc.2014.01.002.

[19] Z.H. Zhao, Y.Z. Lin and J. Niu. Convergence order of the reproducing kernel method for solving boundary value problems. Mathematical Modelling and Analysis, 21(4):466-477, 2016. https://doi.org/10.3846/13926292.2016.1183240. 